\title{
Uso e relevância em abordagem quantitativa de pesquisas em Turismo no Programa de Pós-Graduação em Turismo (PPGTUR-UFRN)
}

Use and relevance in a quantitative approach to research in Tourism in the Graduate Program in Tourism (PPGTURUFRN)

\section{Ayslane Costa da Silva}

Doutoranda pelo Programa de Pós-gradação em Turismo (PPGTUR) da Universidade Federal do Rio Grande do Norte - UFRN, Natal/RN, Brasil

E-mail: ayslanecosta@gmail.com

José Enéas Montenegro Dutra

Professor do Departamento de Turismo da Universidade Federal do Rio Grande do Norte UFRN, Natal/RN, Brasil

E-mail: jeneasdutra@icloud.com

Lidenilza Soares de Lima

Colaboradora do Grupo de Pesquisa Organização de Aprendizagens e Saberes em Iniciativas Solidárias e Estudos do terceiro Setor - OASIS da Universidade Federal do Rio Grande do Norte - UFRN, Natal/RN, Brasil

E-mail: lidenilza@hotmail.com

Mauro Lemuel de Oliveira Alexandre

Professor Associado da Universidade Federal do Rio Grande do Norte - UFRN, Natal/RN, Brasil

E-mail: mauro_alx@yahoo.com

Artigo recebido em: 27-02-2019

Artigo aprovado em: 29-01-2020 


\section{RESUMO}

O desenvolvimento de pesquisas no campo do turismo, possibilita o aprimoramento do conhecimento deste fenômeno. Este trabalho acadêmico visa analisar peculiaridades de pesquisas quantitativas em estudos de turismo. $\mathrm{O}$ artigo tem como objetivo perceber a relevância em abordagem quantitativa de pesquisas em turismo do programa de pósgraduação em turismo da UFRN. A análise de 19 dissertações de mestrado disponíveis nos repositórios da UFRN, possibilitou uma reflexão sobre as abordagens quantitativas adotadas pelos discentes do programa (PPGTUR-UFRN). O trabalho tem ênfase qualitativa, utilizou-se pesquisa bibliográfica e análise de conteúdo das dissertações com auxílio do software IRAMUTEQ. Os resultados do estudo revelaram que a pesquisa quantitativa em turismo constroem-se a partir de um conjunto de técnica de pesquisa e análise bem definidas e que podem contribuir para a compreensão funcionalista do turismo no aperfeiçoamento das técnicas de pesquisa em diálogo com realidade nos locais de pesquisa.

Palavras-chave: Abordagem Quantitativa. Turismo. Fenômenos Turísticos.

\section{ABSTRACT}

The development of research in the field of tourism enable the improvement of knowledge of the phenomenon. This work aims to analyze the peculiarities of quantitative research in tourism studies. The article focus on evaluate the quantitative of tourism research in UFRN's postgraduate tourism program. The analysis of the 19 master's dissertations available in the UFRN repositories allowed a reflection on the research techniques adopted by the students of the program (PPGTUR-UFRN). The work has a qualitative emphasis, the bibliographical research and the content analysis of the dissertations are used with the help of THE software IRAMUTEQ. The results of the study revealed that the quantitative research in tourism was constructed from a set of well-defined research and analysis techniques that can contribute to the functional realization of tourism without the improvement of the research techniques in reality in the research sites.

Keywords: Quantitative Approach. Tourism. Tourism Phenomena. 


\section{INTRODUÇÃO}

O campo de estudos do turismo é bastante complexo e requer vários métodos e disciplinas que possam contribuir para produzir conhecimentos, visando uma maior compreensão do fenômeno (Korstanje, 2015 como citado em Marujo, 2016). Existe atualmente, um quadro geral de métodos e técnicas, que são utilizadas nas pesquisas turísticas, com uma infinidade de procedimentos metodológicos que variam conforme o estudo que se desenvolve (Rejowski, 2007).

Desse modo, em fenômenos turísticos, o grau de probabilidade de recorrência futura, deve-se perceber conforme o grau de constância futura ou à falta de variabilidade dos sucessos passados, neste aspecto, o uso da estatística é necessário (Centeno, 2003).

O Turismo ao se relacionar com diversos ramos de atividade em que todos se relacionam entre si direta ou indiretamente, investigando o que está acontecendo nos demais subsistemas pode ser vital para o funcionamento do sistema global, sendo que, os resultados obtidos nas pesquisas auxiliam neste sentido (Sakata, 2002).

O campo de estudo do turismo, se entrelaça em diferentes disciplinas, possibilitando o estabelecimento de conhecimentos de natureza interdisciplinar, dentro do campo científico (Dencker, 2007).

A produção acadêmica em turismo em nosso país, necessita construção e definição de padrões de qualidade, visando alcançar níveis superiores de contribuição para o desenvolvimento do estado da arte deste campo de estudo acadêmico (Freitag, Tiscoski, \& Neto, 2010).

Para Rejowski (2010) em análise sobre a produção científica em turismo, referentes no exterior e no Brasil, ficou evidenciado que trabalhos sobre o tema publicados no exterior, revela o interesse de pesquisadores pela compreensão da evolução do conhecimento turístico, baseados em pesquisas quantitativas e a preferência pelos periódicos científicos enquanto objeto de estudo, com destaque para o Annals of Tourism Research, reconhecido como o mais importante veículo de comunicação científica da área. Desse modo, o presente trabalho busca responder o questionamento: de que modo se estabelece o uso e relevância da abordagem quantitativa na pesquisa acadêmica em turismo? Com isso, objetiva-se analisar a importância do método do estudo quantitativo em turismo. Assim, esse estudo é relevante, pois busca refletir sobre pesquisas quantitativas no campo de estudos do turismo, visando uma perspectiva crítica, em relação aos caminhos metodológicos sobre esta temática. O objetivo visa analisar o uso dos instrumentos de coleta de dados das pesquisas quantitativas, principais 
técnicas de coleta e análise, do Programa Pós-Graduação em Turismo (PPGTUR) da Universidade Federal do Rio Grande do Norte (UFRN).

Em relação à estrutura do trabalho, este está dividido em quatro seções além desta introdução: a segunda seção traz a revisão de literatura; a terceira seção fala das decisões e os procedimentos metodológicos junto com as descrições da amostra e resultados da pesquisa; na quarta parte, têm-se os resultados destes estudos; e, na quinta parte, as considerações finais do estudo, com suas implicações, limitações e recomendações para futuras pesquisas.

\section{REFERENCIAL TEÓRICO}

\subsection{Abordagem quantitativa}

As abordagens metodológicas se classificam em dois grupos distintos - o quantitativo e o qualitativo. Neste item aprofundaremos a discussão sobre a pesquisa quantitativa.

Inicialmente, a pesquisa quantitativa foi desenvolvida para uso no campo dos estudos de agricultura e das ciências puras, que adotavam uma filosofia positivista do conhecimento que destacava a objetividade e a quantificação dos fenômenos. Ou seja, esta atribuição devese ao fato de que os defensores do paradigma de pesquisa quantitativo entendem que as observações sociais devem ser tratadas como entidades de maneira similar à forma com que os cientistas tratam os fenômenos físicos, e que o observador está separado das entidades que estão sujeitas à observação. De acordo com Johnson, Onwuegbuzie, Tucker e Icenogle (2014) a validade e a confiabilidade dos resultados nas pesquisas produzidas em ciências sociais depende de uma investigação objetiva e livre de generalizações referente ao tempo e contexto.

Os mesmos autores citados anteriormente afirmam que os teóricos da abordagem qualitativa rejeitam o chamado positivismo e defendem a superioridade do construtivismo, idealismo, relativismo, humanismo, hermenêutica e, em alguns casos, pós-modernismo.

O positivismo é uma das doutrinas filosóficas derivadas do iluminismo, tornou-se uma escola filosófica através de Augusto Comte (1798-1857) que era formado em noções de matemática. Entre 1852 e 1854, foi editado seu trabalho conclusivo que foi o Système de politique positive, em plena maturidade intelectual (Penna, 2018).

Segundo o positivismo, a lógica e a matemática seriam válidas por estabelecerem as regras da linguagem, constituindo-se um conhecimento a priori, independente da experiência. No início da década de 60, o debate iniciado pelo filósofo Thomas Kuhn (1922-1996), através da publicação a "Estrutura das revoluções científicas" afeta a maneira de ver a ciência e seu 
método, contribuindo para o esgotamento do "paradigma positivista" (Terence \& Filho, 2006).

Dessa maneira, as pesquisas qualitativas seriam classificadas como interpretativistas e pesquisas com abordagem quantitativas seriam positivistas ou funcionalistas. Assim, a origem do quantitativismo está associada à filosofia da ciência, com Galileu e Newton, e está presente na linha de pensamento empirista e positivista.

Toda pesquisa deve nascer com a formulação de um problema. Portanto, é indispensável que haja uma teoria prévia, ou seja, uma revisão da literatura e as mensurações, para que se formulem o teste de hipóteses, o que permite uma objetividade, ainda que relativa, posto que a escolha do tema de pesquisa e da abordagem teórica nunca é neutra (Ramos, 2013) e para que uso dos métodos quantitativos funcionem corretamente.

Destaca-se que a pesquisa de delineamento quantitativo pressupõe a definição do papel de cada variável e a relação entre elas, gerando hipóteses a serem testadas durante a análise dos dados coletados (Gabriel, 2014).

Dessa forma através dos recursos da informática a quantificação tem sido bemsucedida, conforme Malhotra (2001) já que o objetivo final é 'quantificar' os dados para generalizar os resultados de uma amostra representativa. Assim, a escolha da análise estatística ou a técnica multivariada devem e podem seguir alguns cuidados para ser tomados antes, durante e depois da escrita do relatório de pesquisa para que minimizem os efeitos nocivos da má utilização e que por uma interpretação errônea prejudiquem os resultados.

\subsection{A Pesquisa quantitativa em Turismo}

A partir da Segunda Guerra Mundial é possível verificar que existe um domínio dos métodos quantitativos e, como resultado disso, a investigação qualitativa ficou reduzida à responsabilidade de criar hipóteses que depois serão testadas e validadas por modelos e métodos quantitativos (Walle, 1997). Segundo Peres (2008) isso se deu em contraposição as abordagens filosóficas e históricas e o aparecimento do comportamentalismo. Desde então, passou-se a ter maior preocupação com a objetividade e com as generalizações indutivas, elementos diretamente relacionados com um material empírico, com a quantificação e com métodos sistemáticos e diferenciais. Assim, o turismo sendo uma prática antiga, mas só aparece como área cientifica de estudos recentemente, e sua evolução foi notável, levando-se em conta o curto período de sua ocorrência. Considerando as bases de seu estudo foram 
assentadas antes da segunda Guerra Mundial, seu desenvolvimento científico só ocorreu após a mesma (Rejowski, 1999).

De acordo com Aguiar (2001) em um exame realizado de quarenta anos de publicações em Ciências Sociais no Brasil, efetuado nas principais revistas científicas, incluindo-se nestas a "Revista Brasileira de Ciências Sociais", revela a pequena atenção que, aos métodos quantitativos em Ciências Sociais, uma situação que apenas agora começa a ser corrigida. Para o autor, o declínio das Ciências Sociais Quantitativas no Brasil entra em contraste com a crescente demanda de professores de Ciências Sociais e de estudantes de pósgraduação que estão efetuando uma procura constante de atualização nesses métodos, como forma de elaboração de pesquisas com forte base empírica. Aguiar (2001) afirma que (pp. 23):

Talvez pela redução do volume de recursos para a pesquisa, as Ciências Sociais vêm
apresentando uma tendência marcada para a elaboração teórica de seus objetos de
investigação, sem a mesma preocupação com a documentação das hipóteses
levantadas pelo trabalho teórico. A preocupação com métodos de documentação
quantitativos ficou equacionada como sendo uma perspectiva americanista das
Ciências Sociais. [...\} Pode-se dizer que o emprego de Metodologias Qualitativas se
tornou o método dominante nas Ciências Sociais Brasileiras, e o desenvolvimento de
Métodos Quantitativos assumiu um caráter minoritário, sendo até mesmo objeto de
preconceito.

Ainda para Aguiar (2001), as pesquisas com base quantitativa no Brasil vêm sendo utilizadas por um restrito grupo de pesquisadores em ciências sociais, que encontram dificuldades em formar as novas gerações de profissionais nessa área. Quando se observa o conjunto de publicações brasileiras indexadas no Índice de Ciências Sociais (p. 3).

Num total de 74 títulos, chama a atenção que de 1957 a 2000, isto é, num período de 43 anos, dos 9118 artigos que compõem o índice, apenas 709 correspondem a textos que utilizam Métodos Quantitativos, como recurso de investigação. Por ano, em média, para cada 300 artigos publicados e indexados, cerca de $6 \%$ dos artigos são quantitativos.

Em sua dissertação de mestrado Sakata (2002) aborda o tema sobre as tendências metodológicas da pesquisa acadêmica em turismo. O estudo foi realizado no total de 121 títulos de dissertações e teses de doutorados em turismo, sendo 61 da Universidade de São Paulo - USP, 24 da Universidade Ibero-Americana - UNIBERO e 36 da Universidade Vale do Itajaí - UNIVALI, com o objetivo de conhecer as metodologias mais utilizadas nas pósgraduações em turismo no Brasil. Dentre os resultados deste estudo, destacam-se os seguintes: quanto ao paradigma da pesquisa nota-se que a pesquisa exploratória foi a mais utilizada, seguido por descritivo, explicativo e Histórico. Quanto as técnicas de pesquisa, a mais 
utilizada é a pesquisa de campo, seguida de estudo de caso, depois Bibliográficas e Histórica.

Nas Teses de Doutorado, [...] o Paradigma da Pesquisa, mais utilizado foi o Explicativo, seguido do Teórico, Exploratório-Descritivo e Exploratório. [...] Quanto à Estratégia de Pesquisa, duas que não figuram na lista dos quatro mais utilizados das Dissertações, aparecem aqui em destaque [...], a Fenomenologia [...] e a Etnografia [...]. (Sakata, 2002).

As pesquisas realizadas são em grande parte qualitativa. Poucas dissertações utilizamse do método quantitativo, onde a análise e o tratamento estatístico são necessários. Quanto ao método de coleta de dados Sakata (2002 p. 59) destaca os mais utilizados:

\begin{abstract}
A análise de documentos e registro como fontes de dados foi o mais utilizado, aparecendo em 42 trabalhos (41\%); O questionário foi utilizado em 26 vezes (25\%); O formulário apareceu 25 vezes (24\%) e o método de observação aparece 16 vezes $(15 \%)$. O método de análise a maioria é do tipo expositiva [...] uma vez que o paradigma mais utilizado foi o exploratório.
\end{abstract}

Eidt (2004) realizou um estudo que trata sobre a produção do conhecimento na área de turismo. Tendo como objeto de estudo os artigos científicos publicados na revista "Turismo em Análise" entre os anos de 1995 e 2002, buscando demostrar temas, objetos e metodologias. No total de 122 artigos apenas 54 apresentaram referências metodológicas (p. 47):

\footnotetext{
A pesquisa qualitativa está presente em 43 artigos $(79,63 \%)$ do total de artigos pesquisados. A abordagem quantitativa foi encontrada em sete artigos $(12,97)$. A pesquisa que conjuga ambas modalidades qualitativa/quantitativa está representada com quatro artigos $(7,40 \%)$.
}

Ainda com foco na avaliação da produção cientifica em turismo, Hocayen-da-Silva e Gândara (2007) investigaram trabalhos sobre marketing, qualidade e sustentabilidade. Foram analisados 95 artigos publicados nos anais do ETBL - Encontro Nacional de Turismo de Base Local, entre 2004 e 2005. Observou-se que o método mais utilizado são os estudos qualitativos (77,92\%), nos quais não são utilizados técnicas e instrumentos de análise e coleta de dados apoiados estatisticamente. "A concentração de trabalhos qualitativos pode indicar a fragilidade de pesquisadores em relação aos instrumentos estatísticos que conduzem as pesquisas de caráter quantitativo" (Hocayen-da-Silva e Gandara, 2007, p. 10).

Segundo os autores, dentre as diferentes estratégias qualitativas de pesquisa utilizadas, verificou-se a primazia dos estudos de caso (90\% dos artigos analisados), seguido da estratégia de pesquisa documental em $10 \%$ dos artigos. A estratégias quantitativas de pesquisa 
corresponde a $(22,08 \%)$ destacam-se as surveys como mais utilizadas pelos pesquisadores, representando $88,24 \%$ dos estudos quantitativos.

Outro estudo que aborda a produção científica em turismo foi realizado por Costa e Ferreira (2016) especificamente sobre o tema: eventos corporativos e turismo de negócios em artigos publicados no seminário da Associação Nacional de Pós-graduação em Turismo ANPTUR nos anos de 2005 a 2014. Resultados apontam que do total de 1548, apenas 65 pesquisas referem-se ao turismo de eventos $(4,19 \%)$ somente 11 artigos explanam a questão do turismo de eventos corporativos ou turismo de negócios. Quanto a abordagem (p. 8):

Existe a predominância de trabalhos com a utilização de abordagem qualitativa, com a representação de $63,63 \%$ das pesquisas, enquanto a abordagem quantitativa teve a preferência de $36,36 \%$. Todas as pesquisas têm caráter empírico.

Percebe-se que a partir dos estudos referenciados, a pesquisa de abordagem qualitativa tem predominância nos estudos acadêmicos em turismo nos mais diversos temas investigados como fora apresentado. Em função disso, verifica-se a importância de versar sobre o uso e relevância da pesquisa quantitativa nos campos de pesquisa em turismo afim de colaborar para o melhor entendimento de suas aplicações, contextos e contribuições.

\section{METODOLOGIA}

A natureza da vertente metodológica desta pesquisa foi bibliográfica e documental, com análise de conteúdo, pois, descreve, compreende e explica, não se utilizando de dados estatísticos para analisar o problema. O estudo caracteriza-se por ser de natureza descritiva, a partir do método indutivo, com abordagem qualitativa. Os procedimentos metodológicos adotados foram a pesquisa documental e eletrônicas e, como técnica de análise, adotou-se a análise de conteúdo por adequar-se ao objetivo do trabalho.

Para Köche (1997, p. 122) o objetivo da pesquisa bibliográfica, é "conhecer e analisar as principais contribuições teóricas existentes sobre um determinado tema ou problema, tornando-se instrumento indispensável a qualquer tipo de pesquisa". Com isso, no trabalho adotou-se como fonte de dados utilizada para a confecção deste estudo acadêmico trabalhos concluídos a partir de 2012, no âmbito do PPGTUR, disponíveis no repositório institucional, visando a respectiva análise de conteúdo. O material selecionado foi extraído a partir do critério de escolha de análise adotado no estudo. Na análise dos 19 trabalhos selecionados, com foco em abordagem quantitativa de pesquisas em turismo no (PPGTUR-UFRN), a amostra foi restrita às dissertações de mestrado, publicadas a partir do ano de 2012, 
disponibilizadas no acervo digital e físico da UFRN. A amostra é significativa, pois atende as necessidades deste estudo.

A aplicação da análise de conteúdo, deu-se em decorrência das dissertações selecionadas, que optaram pela abordagem de pesquisa quantitativa e seus respectivos instrumentos de coleta de dados. A partir da percepção do uso de instrumentos de coleta de dados quantitativos, percebeu-se o processo de produção científica em turismo nos trabalhos pesquisados.

Utilizou-se um processo de racionalização dos dados, visando uma melhor compreensão de conceitos a respeito das dissertações de mestrado analisadas, através do uso do software IRAMUTEQ.

O IRAMUTEQ permite identificar as classes de significado, nomeadamente com base nas palavras mais significativas, que representam as ideias e temas dominantes do corpo de dados. O software também emprega o método Reinert, que permite realizar uma classificação de termos; mais especificamente, ele executa uma análise de correspondência fatorial que desconstrói o texto original e o reorganiza com base na repetição e sequência de palavras. $\mathrm{O}$ software viabiliza desde a aplicação de procedimentos simples, como a lexicografia básica realizada pela lematização e cálculo de frequência de palavras, até análises multivariadas, como a CHD - Classificação Hierárquica Descendente que representa num plano cartesiano as diferentes palavras e variáveis associadas a cada uma das classes da CHD.

A análise das revisões envolveu a frequência de computação e as estatísticas de proximidade das palavras utilizadas no corpo do texto e foi realizada utilizando o software IRAMUTEQ (versão 0.7 alpha 2). Referida como lexicometria, ou simplesmente análise de texto, tal análise pode ser conduzida com um número de métodos para reorganizar sequências de texto e criar estatísticas em palavras usando segmentação (Salem, 1986). Para Reinert (1983), o estudo das repetições em um discurso permite tornar-se consciente de tendências ideológicas, zonas de conflito, rupturas, reaproximações ou oposições, que ele denominou "mundos lexicais".

O uso do software IRAMUTEQ requer a organização dos dados das avaliações selecionadas de acordo com variáveis específicas. Nesta pesquisa, as categorias de análise foram predeterminadas a partir da produção da pesquisa quantitativa presentes na literatura. Após inserir os dados, o software realizou uma análise automática e reorganizou-o com base na repetição e sequência de palavras, gerando um resultado que exigiu interpretação adicional pelo pesquisador. Portanto, o conjunto de palavras chave e os resumos das dissertações, foram 
explorados por meio da ferramenta análise de texto, representados por ilustrações e agrupamentos de palavras.

Nesta pesquisa, frente às considerações aqui procedidas, o quadro abaixo direciona as categorias de análise da pesquisa quantitativa adotadas para análise do uso e relevância em abordagem quantitativa na pesquisa em turismo.

Quadro 1 - Categorias orientadoras da pesquisa

\begin{tabular}{|c|c|c|}
\hline Categoria de Análise & Variáveis de Análise & Autores $^{1}$ \\
\hline Contexto de Pesquisa & $\begin{array}{l}\text { Social } \\
\text { Científica }\end{array}$ & Fonseca (2002) \\
\hline Objetivo de Pesquisa & $\begin{array}{l}\text { Natureza do trabalho } \\
\text { Tipo de Problema }\end{array}$ & Oliveira (2011) \\
\hline Universo de Pesquisa & População amostral & Vergara (1997). \\
\hline \multirow{8}{*}{ Método de Pesquisa } & Procedimento & \multirow{4}{*}{$\begin{array}{l}\text { Dwyer \& Seetaram (2012); Brunt, } \\
\text { Horner \& Semley (2017) }\end{array}$} \\
\hline & Etapas/ Phases Stages & \\
\hline & Software & \\
\hline & Estatística & \\
\hline & Instrumento de Coleta & $\begin{array}{l}\text { Dwyer \& Seetaram (2012); Brunt, } \\
\text { Horner \& Semley (2017); Gorard, } \\
\text { (2003); }\end{array}$ \\
\hline & Tipo de Abordagem & $\begin{array}{l}\text { Gorard, S. (2003); Brunt, Horner \& } \\
\text { Semley (2017) }\end{array}$ \\
\hline & Hipótese & $\begin{array}{l}\text { Creswell (2008); Creswell (1994); } \\
\text { Brunt, Horner \& Semley (2017) }\end{array}$ \\
\hline & Técnicas & Brunt, Horner \& Semley (2017) \\
\hline Resultado de Pesquisa & $\begin{array}{l}\text { Descrição dos resultados } \\
\text { Interpretação dos Resultados }\end{array}$ & Brunt, Horner \& Semley (2017) \\
\hline
\end{tabular}

Fonte: Dados da pesquisa, 2018.

No trabalho, a análise dos dados se orienta a partir das categorias acima estabelecidas com uso de análise de conteúdo, seguindo as etapas de pré-análise, exploração do material e tratamento dos dados.

\section{ANÁLISE DOS RESULTADOS}

O corpus (unificado) derivado dos resumos das dissertações possibilitou a estatística textual oferecida pelo IRAMUTEQ. Os 19 textos construídos a partir dos resumos das

\footnotetext{
${ }^{1}$ Autores utilizados e não citados no artigo:

- Creswell, J. W. (1994). Research Design: Qualitative and Quantitative Approaches. Thousand Oaks. CA: Sage

- $\quad$ Creswell, J. (2008). Research design: qualitative, quantitative, and mixed method approaches. (2a. Ed.). London: Sage Publications.

- Dwyer, L., Gill, A., \& Seetaram, N. (Eds.). (2012). Handbook of research methods in tourism: Quantitative and qualitative approaches. Edward Elgar Publishing.

- $\quad$ Fonseca, J. J. S. (2002). Metodologia da Pesquisa Científica. Universidade Estadual do Ceará.

- Oliveira, M. F. (2011). Metodologia científica: um manual para a realização de pesquisas em Administração. Universidade Federal de Goiás, Catalão, GO, Brasil.

- Vergara, S. C. (1997). Métodos de pesquisa em Administração. São Paulo: Atlas.
} 
dissertações foram separados em 107 segmentos de texto (ST), correspondente a 70,86\% de retenção, o que justifica o corpus representativo como aproveitamento de, no mínimo, em $70 \%$ dos segmentos de texto na análise de classificação estatística do IRAMUTEQ.

É válido destacar sobre os termos mais frequentes nos resumos de dissertações selecionados para pesquisa, assim ocorre a prevalência dos termos "turismo", "pesquisa", "turístico", "modelo quantitativo" nas dissertações. Por outro lado, em menor proporção, percebe-se a frequência dos termos "hospedagem", "serviço", "destino", "informação" e "hospedagem".

Assim, o software forneceu o comportamento de frequência das palavras no corpus avaliado, conforme os resumos das dissertações analisadas.

A análise textual no IRAMUTEQ viabilizou os seguintes procedimentos: análises de estatísticas textuais, especificidades e Análise Fatorial de Correspondência (AFC); Classificação Hierárquica Decendente (CHD); Análise de Similitude; nuvem de palavras. A natureza do grupo de palavras gerou a distribuição de classes seguindo o critério de maior frequência e inter-relação.

Na Classificação Hierárquica Descendente (CHD), o conteúdo processado gerou cinco classes: Classe 1 com 25 STs (23,36\%); Classe 2 com 15 STs (14,02\%); Classe 3 com 25 STs (23,36\%); Classe 4 com 17 STs (15,89\%) e Classe 5 com 25 STs (23,36\%). A Figura 1 abaixo mostra o primeiro dendograma gerado pelo IRAMUTEQ que revela o relacionamento das classes dentro do corpus. As classes 4 e 5, denominadas contexto e resultados de pesquisa respectivamente, formam o subcorpus A, a classe 3 abrange o universo de pesquisa, a classe 2 caracteriza o objeto da pesquisa e, a classe 1, sendo a mais representativa expressa a metodologia adotada nas pesquisas em turismo no (PPGTUR/UFRN). 
Figura 1 - Dendograma da Classificação Hierárquica Descendente

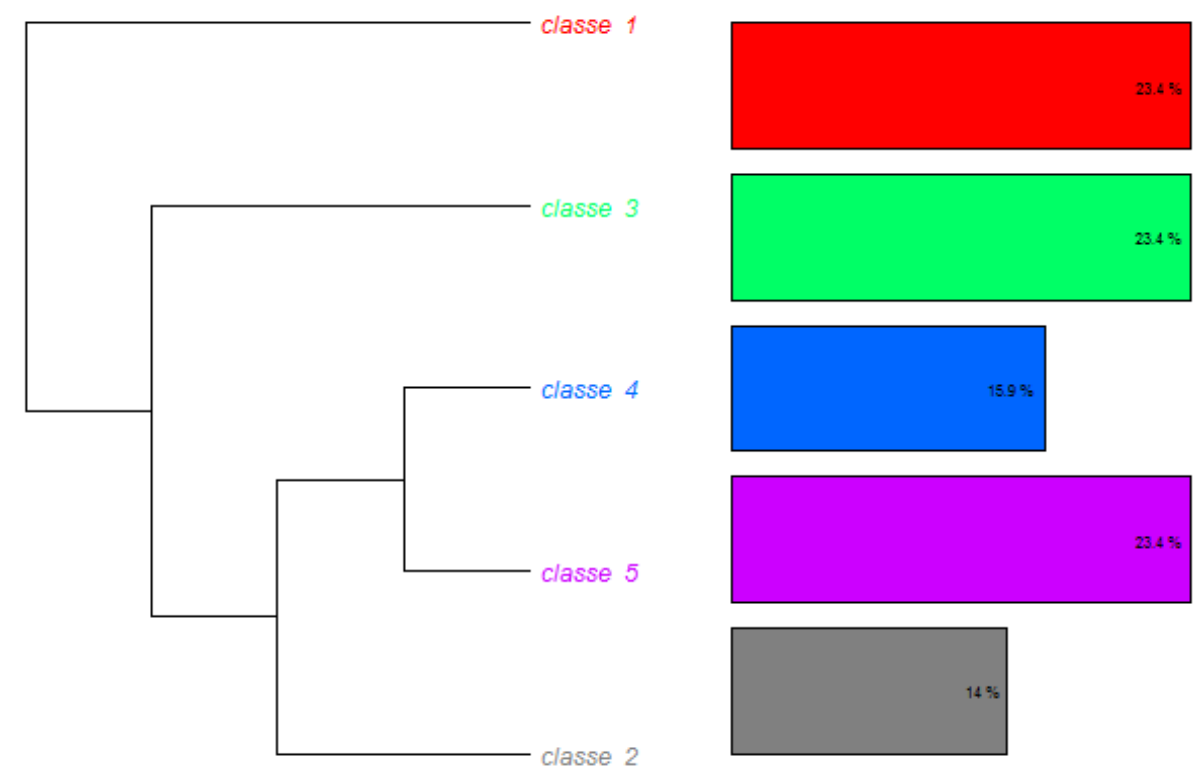

Fonte: Software IRAMUTEQ, dados da pesquisa, 2018.

A Figura 2 abaixo, representa um organograma gerado pelo IRAMUTEQ, em são identificadas as divisões realizadas no tratamento do conteúdo do corpus, visando o agrupamento em classes finais.

Figura 2 - Organograma das classes, frequências e $\mathrm{x}^{2}$ 


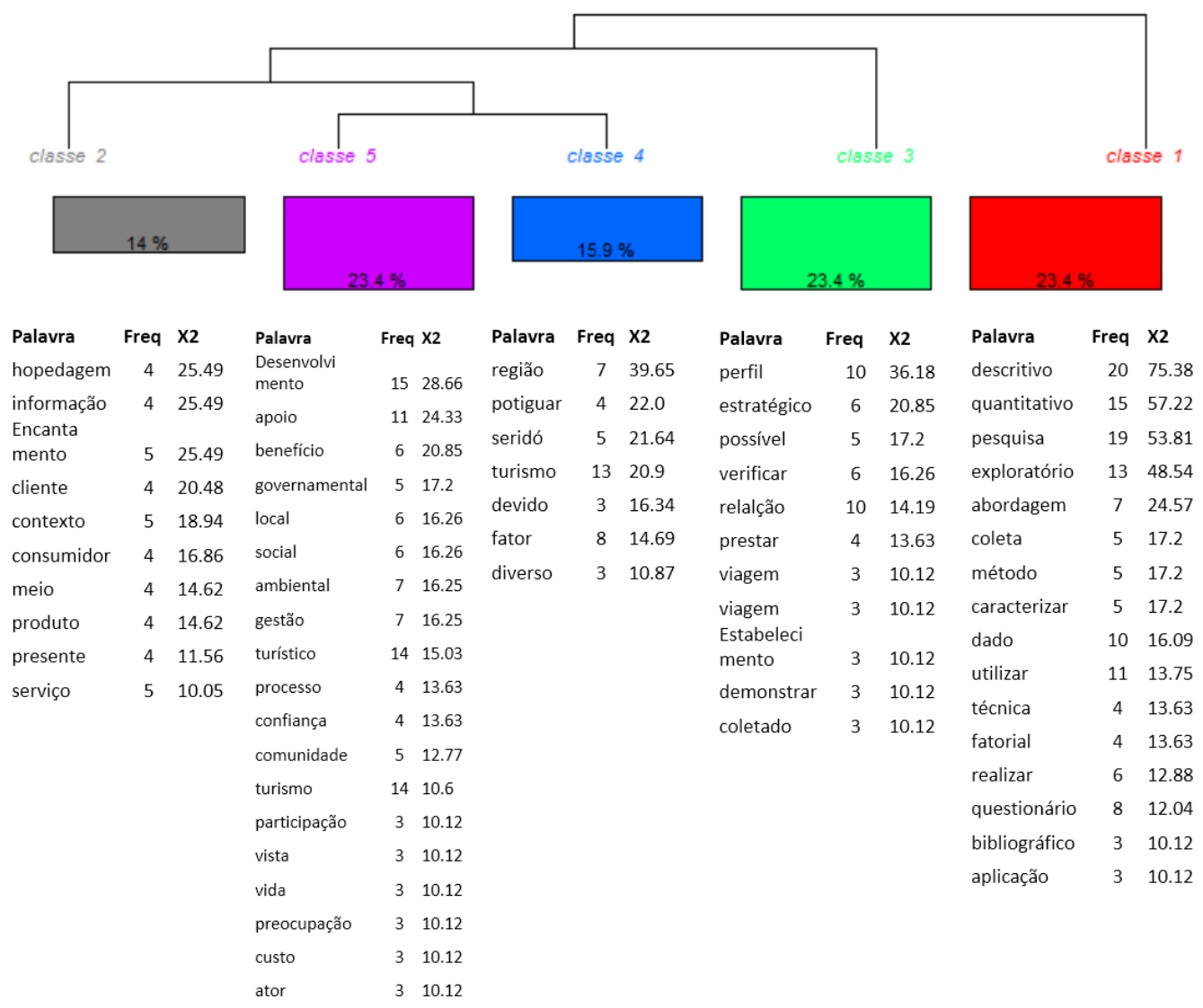

Fonte: Software IRAMUTEQ, dados da pesquisa, 2018.

Desse modo, as formas do corpus foram agrupadas em cinco classes estáveis, ou seja, conforme os segmentos dos resumos com vocabulários semelhantes. Assim, o organograma busca identificar o conteúdo lexical de cada umas das cinco classes. Na classe 1 foram agrupados temos relacionados a "descritivo", "quantitativo" e "pesquisa". Na classe 2 são relacionados os termos referentes ao objetivo da pesquisa onde termos como "hospedagem", "informação", "encanta" aparecem mais expressivamente. Na classe 3 são visualizados os termos relativos ao universo da pesquisa onde as palavras "perfil", "estratégico" e "possível".

As classes 4 e 5 relacionam termos que expressam contexto e benefícios da pesquisa, onde termos como "desenvolvimento", "apoio", "benefício" e "região", "potiguar" e "Seridó", apontando o campo de pesquisa e sua contextualização social/científica.

Com a análise CHD, onde verifica-se o tipo de relação entre classes, o plano cartesiano a seguir representa a Análise Fatorial de Correspondência (AFC), onde mostra a frequência das palavras no corpus dentro das classes correspondentes.

Figura 3 - Análise Fatorial por Correspondência 


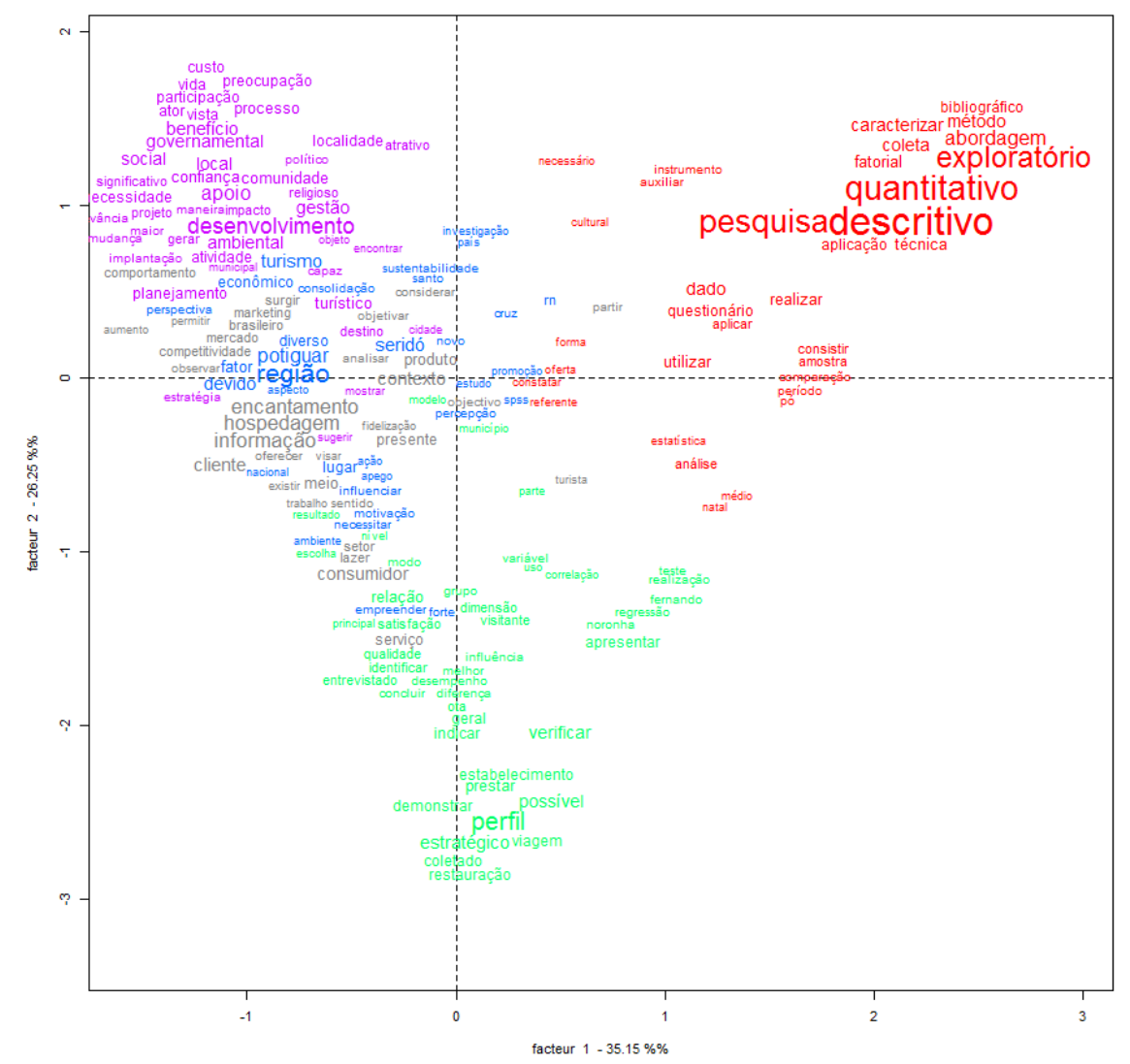

Fonte: Software IRAMUTEQ, dados da pesquisa, 2018.

Diante a representação gráfica na figura 3, observa-se a distribuição dispersa das palavras nos quadrantes, indicando aproximação entre as classes 3, 4 e 5. Sendo assim, a proximidade observada entre as classes que aportam universo, contexto e benefícios da pesquisa estão correlacionadas dentro do corpus, indicando termos afins por posicionarem-se mais próximos ao centro. A denominação das classes a partir da CHD derivou de categorias analíticas construídas de produções teórica do tema, conforme quadro 1. Observa-se também a distribuição das classes 1 e 2 no gráfico, onde estão separadas e dispersas em mais de um quadrante, isto é, os termos contidos nas classes metodologia e objetivo da pesquisa comportam-se distintamente, significativamente distantes das classes 3, 4 e 5. Os termos mais distantes, não são utilizados num mesmo contexto no corpus. Como exemplo, seria o termo "quantitativo", distante e por isso, forma incomum ao termo "perfil", localizado num quadrante distante.

A partir da Análise de Similitude, que permite analisar a relação entre as palavras do corpus, infere-se a construção do texto e a quais outras expressões o termo se relaciona, a partir da coocorrência entre as palavras. A figura 4 expressa o gráfico das palavras e a relação das palavras associadas na classe. Abaixo, na classe Método de Pesquisa, expressões como 
descritivo, quantitativo e pesquisa tem mais destaque na classe 1. Tais expressões apresentam intensa relação entre si, conforme figura 4. Observa-se que, na ramificação da expressão pesquisa, há forte relação entre os termos "utilizar e "realizar" que, pela análise de concordância de classe, apresenta-se como indicativo de métodos de pesquisa, objetivos postulados, instrumentos de coleta e técnicas de análise de dados utilizados nas pesquisas em turismo.

Figura 4 - Análise de Similitude (Gráfico da Classe Método de Pesquisa)

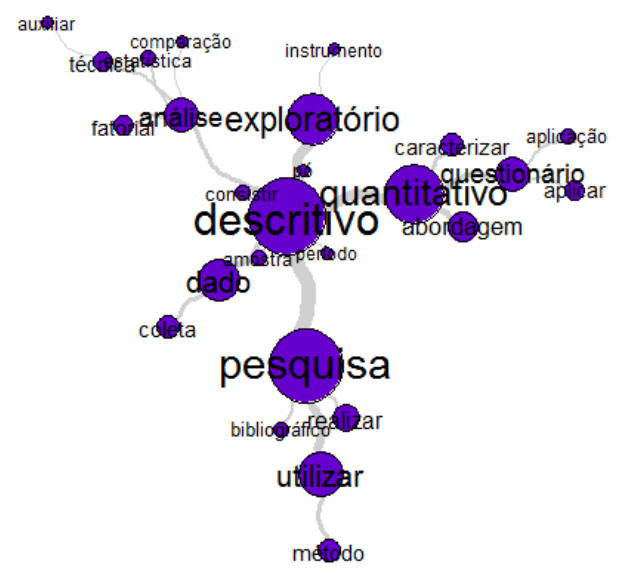

Fonte: Software IRAMUTEQ, dados da pesquisa, 2018.

$\mathrm{Na}$ análise da expressão quantitativo, palavra de segunda maior força na classe (x2=57,22), há relação forte e significativa entre expressões como questionário e aplicação, isto é, nas pesquisas em turismo com abordagem quantitativa, há utilização de questionários estruturados e fechados como instrumentos de coleta de dados da pesquisa. Gorard (2003) analisa as principais metodologias utilizadas na pesquisa de turismo, hotelaria e gestão de eventos, que são usadas para obter informações dos entrevistados (ou seja, pessoas) que respondem perguntas sobre si mesmos, seu conhecimento sobre determinado assunto e suas opiniões. Ao discorrer sobre a pesquisa quantitativa, revela que utiliza-se o questionário de pesquisa como principal instrumento de coleta de dados pois permite a comparação de respostas na análise do fenômeno em estudo.

Quanto ao termo exploratório $(\mathrm{x} 2=48,54)$, relaciona-se de maneira forte e direta com o termo descritivo, e pela análise de concordância na classe, e mostra que estudos descritivosexploratórios são adotados significativamente em pesquisas quantitativas adotado nas produções em turismo no PPGTUR/UFRN. Quanto ao termo análise, as ramificações relacionam-se diretamente com termos como fatorial, estatística e técnica, indicando pela relação de concordância na classe que essa relação expressa uso de técnicas estatísticas 
avançadas de análise de dados nas pesquisas, como análise fatorial, bivariada, multivariada, regressão linear, no software SPSS para análise estatística avançada em ciências sociais.

O gráfico da classe Objeto da Pesquisa, figura 10 abaixo, evidencia termos como encantamento, informação e hospedagem como os mais expressivos na classe. A relação de ramificação com termos como cliente, produto, consumidor e contexto é significativa e forte, e pela análise de concordância, se traduz nos objetos de estudo adotados nos trabalhos de pesquisa em turismo. Esses termos, combinados a análise de similitude, mostram relação forte com as palavras serviço e consumidor. Algumas pesquisas no turismo vêm dando foco ao processo de encantamento e experiência de consumo (Almeida \& Nique, 2007; Crotts \& Magnini, 2011). Em pesquisas quantitativas, estudam-se esses termos aplicados a perspectiva de dimensões estruturadas, buscando relações, inter-relações, de natureza e influência positiva e negativa, diretamente proporcional ou inversamente proporcional às hipóteses elaboradas na investigação do problema de pesquisa.

Figura 5 - Gráfico da Classe Objeto de Pesquisa

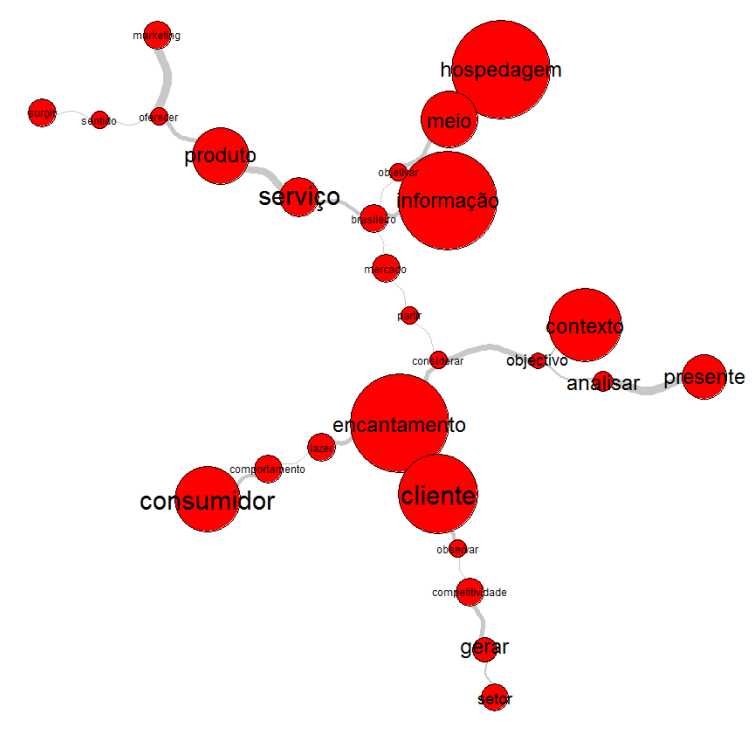

Fonte: Software IRAMUTEQ, dados da pesquisa, 2018.

No gráfico da classe Universo da pesquisa, os termos perfil, estratégico e possível são mais expressivos por serem de maior significância. Há relação forte com expressões como 
relação, satisfação que expressam, pela análise de concordância de classe, comportamento cognitivo do público analisado na pesquisa sob forma de qualidade de serviços, apego e fidelidade. Em pesquisas quantitativas em turismo, tais termos inferem dimensões complexas e de base teórica sólida na literatura. Estudos acerca das relações de influência entre fatores de compra e utilização de produtos e serviços turísticos vem ganhando destaque, pois são apoiados em teorias da psicologia e do marketing na análise do comportamento de compra de consumidores.

Figura 6 - Gráfico da Classe Universo de Pesquisa

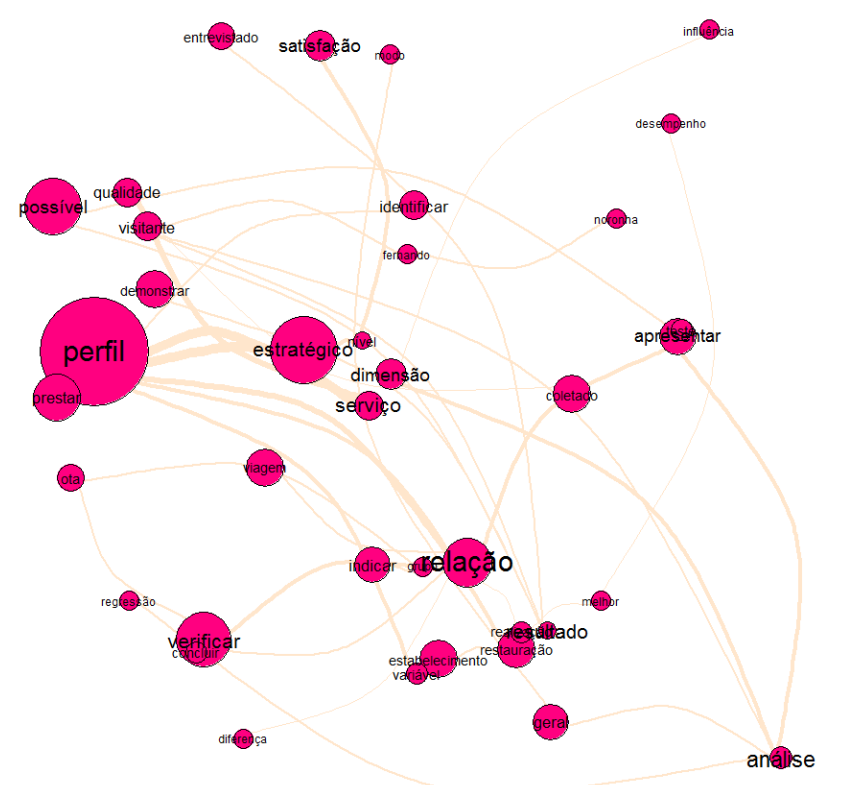

Fonte: Software IRAMUTEQ, dados da pesquisa, 2018. 
$\mathrm{Na}$ análise da classe Contexto da Pesquisa, as expressões região, potiguar e Seridó, ganham destaque na representação gráfica por apresentarem maior incidência na classe dentro do corpus. A representação por comunidades, expressa de forma abstrata e dividida por cores, explica a relação entre as palavras na classe, conforme a Figura 7 abaixo. Percebe-se que as pesquisa quantitativas em turismo no PPGTUR/UFRN tem, como um dos locus principais de pesquisa, a região do Seridó potiguar. Expressões como sustentabilidade aparecem na classe onde relacionam-se com econômico, desenvolvimento e percepção. Pela análise de concordância, o termo infere-se no contexto de desenvolvimento sustentável de destinos turísticos no Rio Grande do Norte, a partir da visão de residentes e frequentadores que vistam as regiões turísticas do estado.

Figura 7 - Gráfico da Classe Contexto de Pesquisa

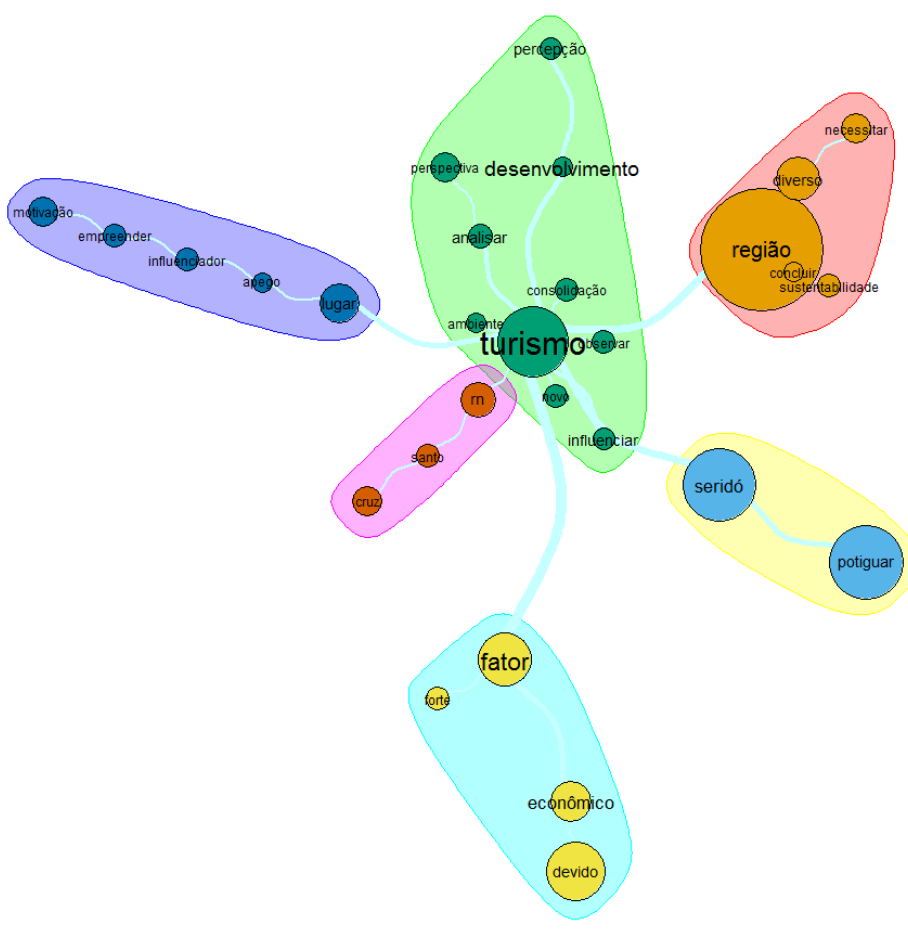


Fonte: Software IRAMUTEQ, dados da pesquisa, 2018.

$\mathrm{Na}$ classe Resultados de pesquisa, os termos mais expressivos na classe são desenvolvimento, apoio e benefício. As ramificações, explicadas pela concordância na classe, revelam os principais resultados observados e os benefícios observados na pesquisa. O termo apoio, como observa-se, tem relação forte com os gestão e social, indicando ações e fases de planejamento municipal de destinos turísticos, participação de stakeholders e gestão de projetos. Pesquisas quantitativas em turismo vêm dando foco para temáticas de gestão e planejamento de destinos. Brunt, Horner e Semley (2017) discorrem que a partir da postura positivista adotada em pesquisas quantitativas, o pesquisador percebe o objeto de pesquisa dentro da perspectiva funcionalista, buscando analisar a representatividade do fenômeno no estudo, com objetividade ao distanciar-se do processo de pesquisa. Por isso, o fenômeno observado na pesquisa tende a produzir resultados dedutivos que possibilitam a tomada de decisão no contexto informado.

Figura 8 - Gráfico da Classe Resultado de Pesquisa 


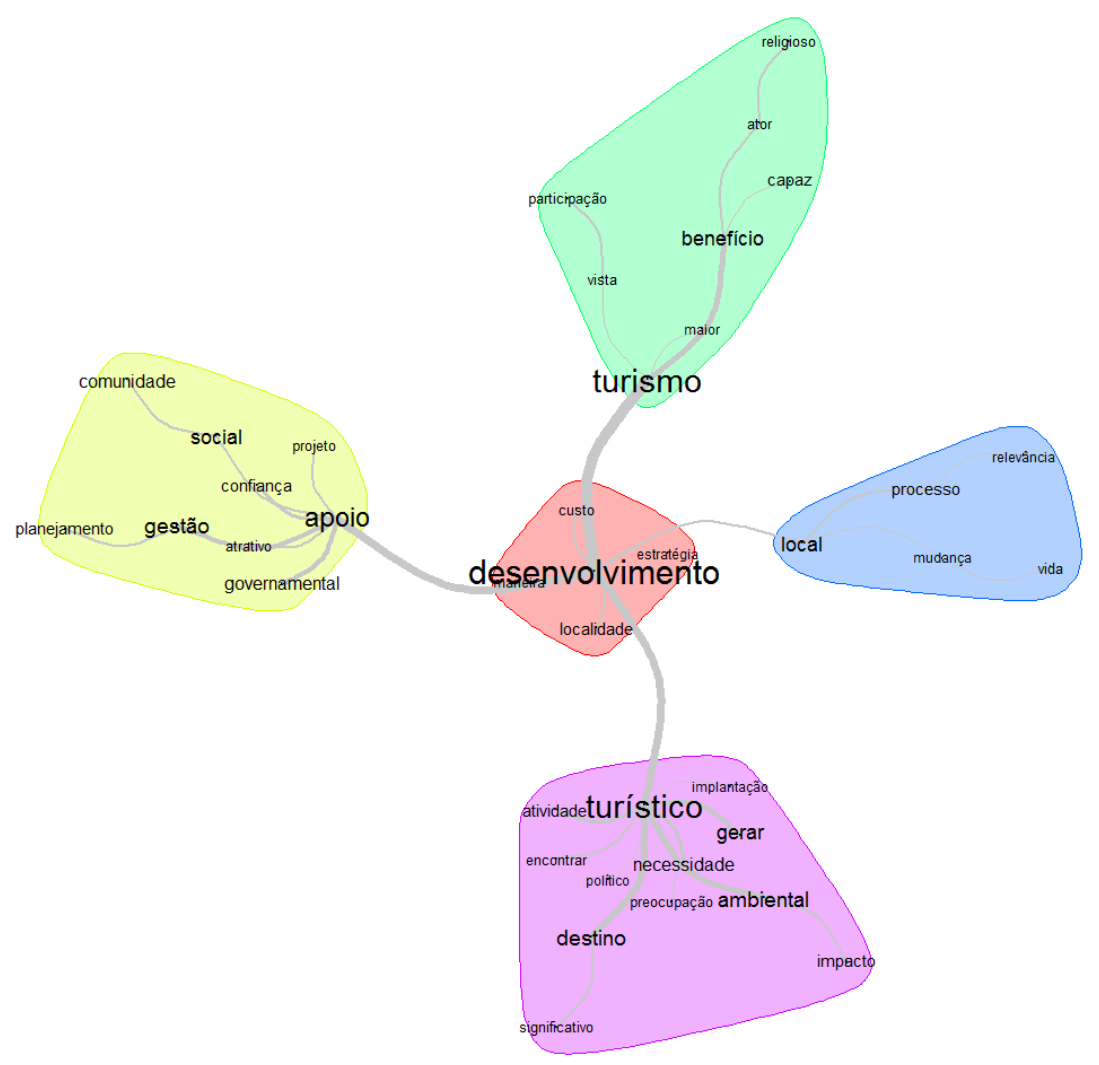

Fonte: Software IRAMUTEQ, dados da pesquisa, 2018.

\section{COSIDERAÇÕES FINAIS}

Pode-se ver ao longo do trabalho a discussão sobre o uso e relevância da abordagem quantitativa em pesquisa no turismo, o que possibilita perspectiva de construção teórica acerca dos métodos de pesquisa já consolidados na produção científica do turismo.

Tendo em vista o que foi estabelecido no trabalho, é possível compreender que as pesquisa quantitativas em turismo constroem-se a partir de um conjunto de técnica de pesquisa e análise bem definidas, com precisão consolidada por métodos estatísticos rígidos. Apesar dos estudos na área serem de maioria qualitativa, a abordagem quantitativa faz-se necessária para representação do fenômeno turístico pela visão positivista funcionalista, em áreas de estudo como planejamento de destinos e percepção dos residentes, por exemplo.

Esse estudo permite uma contribuição importante para a compreensão acerca dos métodos de pesquisa e abordagem quantitativa nas pesquisas em turismo, reunindo estudos seminais e atuais, categorias e variáveis de pesquisa, em campos de atuação na pesquisa acadêmica no turismo e nos contextos em níveis de atuação estratégica, social e cultural. Assim, o estudo alcança seus objetivos, pois fornece um panorama da produção acadêmica em turismo, recortando o uso e relevância científica da abordagem quantitativa em pesquisas no (PPGTUR-UFRN). 
Algumas limitações foram percebidas sobretudo a amostra limitada de dissertações utilizadas na construção do trabalho. Tendo-se uma amostra mais significativa, considerações mais representativas poderiam ter sido observadas a respeito das técnicas estatísticas, instrumentos de pesquisa, uso de software. É relevante frisar que, estudos que utilizam amostragem maiores, em pesquisas científicas, sobretudo, voltadas para o campo do turismo, contribuem para a validação do estudo, aumentando a confiabilidade e abrangência dos achados.

Como indicações temáticas para estudos seguintes, podem ser identificados temáticas que possibilite expandir as discussões entre método quantitativo e qualitativo no turismo, dando espaço para possibilidade de pesquisadores avaliarem o uso de métodos mistos com duas abordagens de pesquisa, combinando técnicas de pesquisa, coleta e análise dos dados da pesquisa.

Portanto, pesquisas que utilizam a abordagem quantitativa em turismo, com suas respectivas análises, contribuem para a validação da amostragem, visando a consolidação dos dados pesquisados, dando consistência na abordagem do objeto pesquisado. Assim, verificouse que a abordagem quantitativa, em estudos desenvolvidos através de pesquisas em turismo no Programa de Pós-graduação em Turismo da UFRN (PPGTUR-UFRN), podem contribuir para uma compreensão (dedutiva, objetiva) e aplicação dos estudos, com relação a significância representativa dos contextos de pesquisa em turismo no estado do Rio Grande do Norte, por região, em função do aperfeiçoamento das técnicas de pesquisa em diálogo com realidade nos locais de pesquisa.

\section{REFERÊNCIAS}

Aguiar, N. (2001). Qual a contribuição dos métodos quantitativos em ciências sociais para o conhecimento da sociedade brasileira? Comunicação Apresentada, (25).

Almeida, S. O., \& Nique, W. M. (2007). Encantamento do cliente: proposição de uma escala para mensuração do constructo. Revista de Administração Contemporânea, 11(4), 109-130.

Brunt, P., Horner, S., \& Semley, N. (2017). Research Methods in Tourism, Hospitality and Events Management. United Kingdom: Sage Publishing.

Centeno, R. R. (2003). Metodologia da Pesquisa Aplicada ao Turismo - Casos Práticos. São Paulo: Roca.

Costa, J. R., \& Ferreira, L. V. F. (2016). Turismo de negócios e eventos corporativos: análise das pesquisas apresentadas no seminário da ANPTUR 2005-2014. Revista Turismo: estudos e práticas, $5(2)$. 
Crotts, J. C., \& Magnini, V. P. (2011). The customer delight construct: Is Surprise Essential? Annals of Tourism Research, 38(2), 719-722.

Dencker, A. F. M. (2007). Pesquisa em Turismo: planejamento, métodos e técnicas. São Paulo: Futura.

Eidt, K. R, G. (2004). Turismo em Análise: a produção do conhecimento na área do turismo. Dissertação de Mestrado em Turismo e Hotelaria, Universidade do Vale do Itajaí, Itajaí, SC, Brasil.

Freitag, B. B., Tiscoski, G. P., \& Neto, L. (2010). Análise da Produção Acadêmica em Administração sobre Turismo. Anais do XXXIV Encontro da EnANPAD. Rio de Janeiro.

Gabriel, M. L. D. (2014). Métodos Quantitativos em Ciências Sociais Sugestões para Elaboração do Relatório de Pesquisa. Desenvolvimento em Questão, 12(28), 348-369.

Gorard, S. (2003). Quantitative methods in social science research. A\&C Black.

Hocayen-da-silva, A., \& Gândara, J. M. G. (2007). Marketing, qualidade e sustentabilidade: análise da produção científica do Encontro Nacional de Turismo de Base Local-ENTBL, nos anos de 2004 e 2005. Anais do IV Seminário da Associação Pacional de Pesquisa e PósGraduação em Turismo. São Paulo: Editora Aleph.

Johnson, R. B., Onwuegbuzie, A. J., Tucker, S. A., \& Icenogle, M. L. (2014). Conducting mixed methods research: Using dialectical pluralism and social psychological strategies. In: Leavy, P. (Ed.). Oxford library of psychology. The Oxford handbook of qualitative research. Oxford University Press.

Koche, J. C. (1997). Fundamentos de Metodologia Científica: teoria da ciência e prática da Pesquisa. Rio de Janeiro: Editora Vozes.

Malhotra, N. (2001). Pesquisa de marketing. Porto Alegre: Bookman.

Marujo, N. (2013). A pesquisa em turismo: reflexões sobre as abordagens qualitativa e quantitativa. 6(14), 1-16.

Penna, L. A. (2018). Positivismo. Recuperado em 05 agosto, 2018, de https://cpdoc.fgv.br/sites/default/files/verbetes/primeira-republica/POSITIVISMO.pdf

Peres, P. (2008). "Comportamento ou instituições? A evolução histórica do neoinstitucionalismo da ciência política". Revista Brasileira de Ciências Sociais, 23 (68), 5371.

Ramos, M. P. (2013). Métodos quantitativos e pesquisa em ciências sociais: lógica e utilidade do uso da quantificação nas explicações dos fenômenos sociais. Mediações: Revista De Ciências Sociais, 18(1), 55-65.

Reinert, M. (1983). Une méthode de classification descendante hiérarchique: application à l'analyse lexicale par contexte. Cahiers de l'analyse des données, 8(2), 187-198.

Rejowski, M. (1999). Turismo como disciplina no pensamento internacional. In: Rejowski, M. Turismo e pesquisa científica. São Paulo: Papirus.

Rejowski, M. (2007). Produção científica em Turismo: análise de estudos referenciais no exterior e no Brasil. (7a. ed.). Campinas, SP: Papirus. 
Rejowski, M. (2010). Produção Científica em Turismo: análise de estudos referenciais no exterior e no Brasil. Revista Turismo em Análise, 21(2), 224-246.

Sakata, M. C. G. (2002). Tendências metodológicas da pesquisa acadêmica em turismo. Tese de Doutorado, Universidade de São Paulo, São Paulo, SP, Brasil.

Salem, A. (1986). Segments répétés et analyse statistique des données textuelles. Histoire \& Mesure, 1, 5-28.

Terence, A. C. F., \& Filho, E. E. (2006). Abordagem quantitativa, qualitativa e a utilização de pesquisa-ação. Anais do XXVI Encontro Nacional de Engenharia de Produção-ENEGEP. Fortaleza, CE, Brasil.

Walle, A. (1997). "Quantitative versus qualitative tourism research". Annals of Tourism Research, 24(3), 524-536.

FORMATO PARA CITAÇÃO DESTE ARTIGO

SILVA, A. C., DUTRA, J. E. M., LIMA, L. S., \& ALEXANDRE, M. L. O. (2020). Uso e relevância em abordagem quantitativa de pesquisas em Turismo no Programa de Pós-Graduação em Turismo (PPGTUR-UFRN). Revista de Turismo Contemporâneo, 8(1), 65-87. https://doi.org/10.21680/2357-8211.2020v8n1ID16970 